\title{
Validação do Questionário de Perfis de Valores (QPV) no Brasil
}

\author{
Alvaro Tamayo \\ Universidade de Brasília \\ Juliana Barreiros Porto ${ }^{1}$ \\ Universidade Católica de Brasília
}

\begin{abstract}
RESUMO - O objetivo desta pesquisa foi validar para o Brasil o Questionário de Perfis de Valores (QPV), desenvolvido por Schwartz. Para a tradução do questionário, utilizou-se a estratégia de tradução-retradução. O QPV foi aplicado a uma amostra de 614 estudantes do Ensino Fundamental, Médio e Superior. Os dados foram analisados por meio da técnica de escalonamento multidimensional em uma estrutura bidimensional. A configuração espacial obtida oferece indicações positivas de validade de construto do instrumento. As regiões encontradas foram: Conformidade, Tradição, Segurança, Estimulação, Universalismo/ Benevolência, Poder/Realização e Autodeterminação/Hedonismo. As três últimas regiões ficaram compostas por tipos motivacionais adjacentes. A convergência de resultados descritivos e comparativos com achados de pesquisas internacionais constitui também um conjunto de elementos importantes de que o instrumento mede o que pretende medir.
\end{abstract}

Palavras-chave: valores básicos; teoria de valores de Schwartz; validação de escala.

\section{Validity of the Portrait Values Questionnaire (PVQ) in Brazil}

\begin{abstract}
The objective of this research was to validate to Brazil the Portrait Values Questionnaire (PVQ) developed by Schwartz. The PVQ was translated into Portuguese using the back-translation procedure and was applied to a sample of 614 students from primary, high and college education. The data were analyzed by means of the multidimensional scaling technique using a bi-dimensional structure. The spatial configuration obtained offers a positive indication for the construct validity of the instrument. The regions found were: Conformity, Tradition, Security, Stimulation, Universality/Benevolence, Power/Achievement and Self-Determination/Hedonism. The last three regions were composed of adjacent motivational types. Descriptive and comparative results converging with international findings also constitute a set of important elements that supports the validity of the instrument.
\end{abstract}

Keywords: basic human values; value theory of Schwartz; scale validation.

A pesquisa relatada neste artigo teve como objetivo principal traduzir para o português e validar o Portrait Values Questionnaire (PVQ), construído por Schwartz e colaboradores (Schwartz, 2005a; Schwartz \& cols., 2001) para avaliação dos valores pessoais. Ao construir o PVQ, os autores tinham como objetivo superar o caráter abstrato dos itens de outros instrumentos de medida dos valores, amplamente utilizados em pesquisa, e, dessa forma, propor uma medida que pudesse ser utilizada com pessoas de baixo nível de escolaridade e com crianças e adolescentes.

Os valores têm sido objeto de estudo desde épocas remotas. O seu estudo científico, porém, começou com as primeiras tentativas de medida. Em 1928, Spranger, na Alemanha, elaborou uma espécie de tipologia para o estudo dos valores e da personalidade. Os tipos propostos pelo autor foram: o Teórico, caracterizado pela procura da verdade; o Político, definido pela busca do poder; o Econômico, focalizado na procura do útil; o Social, constituído fundamentalmente pela busca do amor dos outros; o Estético, caracterizado pelo interesse na avaliação das formas e da harmonia; e o Religioso, definido pela busca da unidade mística com uma força superior. Spranger, entretanto, não elaborou um instrumento para a verificação da sua teoria. Em 1931, o seu estudo foi

1 Endereço para correspondência: Qd. 104 Lt. 04 Ap. 202. Águas Claras, DF. CEP 71909-180. Fone: (61) 3448-7204.E-mail: porto.juliana@ gmail.com. traduzido para o inglês e a sua teoria foi utilizada como base para a construção do primeiro instrumento para avaliação dos valores, denominado Study of Values. Esse instrumento foi elaborado por Allport, Vernon e Lindzey (1951). Posteriormente, Larcebeau (1974), na França, utilizou também a teoria de Spranger para construir o Questionnaire d'Intérêts et de Valeurs (QIV).

Tanto o Study of Values quanto o QIV tiveram um sucesso relativo. O instrumento de Allport e cols. (1951) foi utilizado particularmente em pesquisas para avaliação dos valores e para estudar temas tais como as diferenças na importância atribuída aos valores por diversos grupos profissionais (Allport, Vernon \& Lindzey, 1970) e para predizer a ocupação de alunos de graduação 25 anos mais tarde (Huntley \& Davis, 1983). O instrumento de Larcebeau (1974) foi utilizado exclusivamente na orientação profissional e escolar. O sucesso reduzido desses instrumentos para o estudo dos valores deve-se, em parte, ao fato de que eles avaliam mais os interesses do que os valores. A própria teoria, que está na base dos dois instrumentos, descreve as pessoas a partir dos seus interesses por assuntos econômicos, religiosos, estéticos, sociais, políticos ou teóricos. O foco diferencial nessas categorias de interesses dá origem ao que o autor da teoria (Spranger, 1931) chamava de Lebensformen, isto é, estilos de vida definidos pelas áreas de interesse nas quais o indivíduo circula com maior frequência e satisfação. O instrumento de Allport e colaboradores foi severamente criticado por ser um 
teste de interesses ocupacionais (Duffly, 1940; McLaughlin, 1965). O QIV de Larcebeau (1974) foi destinado, desde o início, à orientação profissional.

Contrariamente aos dois instrumentos acima discutidos, o Rokeach Values Survey (RVS), publicado em 1967, foi amplamente utilizado no estudo dos valores e continua sendo utilizado em diversas pesquisas das ciências sociais. O RVS é um inventário de 36 valores, dividido em duas partes: 18 valores terminais que se referem a metas gerais da existência (e.g., liberdade, igualdade) e 18 valores instrumentais que expressam comportamentos ou qualidades morais (e.g., obediente, honesto). A escala de respostas utilizada é de tipo ordinal. A escala apresenta duas listas de valores (terminais e instrumentais). Para cada lista, o respondente deve colocar em ordem de importância os valores descritos, ficando em primeiro lugar o mais importante e, por último, o menos importante.

Os critérios utilizados por Rokeach (1967) para a construção dos itens terminais e instrumentais não foram os mesmos. Os valores terminais foram identificados a partir da literatura que evocava valores americanos ou de outras sociedades. Além disso, o autor solicitou a um grupo de 30 estudantes de pós-graduação em psicologia que citassem valores e entrevistou uma amostra de 100 adultos sobre os seus valores. Essa amostra era representativa de uma pequena cidade americana. Dessa forma, obteve várias centenas de valores. Rokeach eliminou os sinônimos, os valores muito específicos e aqueles que não representavam metas da existência. Os itens representando os valores instrumentais foram construídos a partir de uma lista de 555 palavras relativas a traços de personalidade, compilada por Anderson (1968). Esse autor utilizou como fonte para a sua lista um levantamento anterior composto por 18.000 palavras-traço compiladas por Allport e Odber em 1936 (citador por Rokeach, 1973). Rokeach (1967) utilizou vários critérios para a escolha dos itens instrumentais a partir das 555 palavras compiladas por Anderson. Primeiramente, eliminou todos os termos negativos, reduzindo, assim, a lista a 200 palavras. Outros critérios de seleção foram: eliminação dos sinônimos e de palavras com significado muito próximo e seleção de termos que expressavam valores americanos ou valores que apresentavam um mesmo sentido em todas as culturas. Dessa forma, ele chegou aos 18 valores instrumentais.

Para validação do instrumento, Rokeach (1973) realizou análise fatorial com rotação varimax, utilizando uma amostra nacional norte-americana composta por 1.489 indivíduos. Os resultados revelaram sete fatores bipolares: gratificação imediata versus gratificação deferida, competência versus moralidade religiosa, auto-restrição versus auto-expansão, orientação social versus orientação pessoal, segurança societal versus segurança familiar, respeito versus amor, centrado em si versus centrado nos outros. Nenhum dos fatores obtidos explicou mais do que $8 \%$ da variância observada. Além da análise com a amostra total, o autor verificou também a estrutura fatorial do instrumento com as seguintes subamostras: brancos, negros, homens e mulheres. Os resultados foram muito semelhantes aos obtidos com a amostra total. Mais uma vez, a variância total explicada ficou em torno de $40 \%$. O autor considerou esses resultados insatisfatórios. Numa nova tentativa de validação, o autor utilizou a SSA (Similarity Structure Analysis). Rokeach não apresenta os resultados, mas afirma que estes são essencialmente os mes- mos que os encontrados com a análise fatorial. Ele interpreta os resultados das suas tentativas de validação do RVS como prova de que os valores que compõem o instrumento são independentes e que, portanto, não podem se integrar para constituir um ou mais fatores. Esse autor afirma: "mais uma vez deve-se concluir que os 36 valores terminais e instrumentais não podem facilmente ser reduzidos a um número menor" (p. 48).

A precisão do RVS foi avaliada ao nível dos dois conjuntos de valores, terminais e instrumentais, e ao nível de cada um dos 36 valores. Foi utilizada a técnica de teste-reteste com intervalo de 4,5 semanas. A precisão foi de 0,80 para os valores terminais e 0,70 para os instrumentais. Rokeach (1973) não fornece os resultados em detalhe para a precisão calculada ao nível de cada um dos valores, mas os sintetiza da seguinte forma: quatro valores instrumentais apresentaram precisão em torno de $0,50,10$, em torno de 0,60 , três, em torno de 0,70 , e um, de 0,88 . A precisão para os valores instrumentais foi um pouco menor, variando entre 0,45 e 0,70. Rokeach comenta que Feather, em 1973, obteve, na Austrália, resultados mais ou menos equivalentes aos seus.

Mais recentemente, têm sido construídos instrumentos para avaliação dos valores a partir de modelos teóricos mais elaborados. Dois modelos merecem ser mencionados: o modelo de Gouveia (2003) e o modelo de Schwartz (1992, 2005b). Os dois fundamentam-se na relação estreita entre valores e necessidades, amplamente defendida por Roheach (1973). Segundo esse autor, "os valores são representações cognitivas e transformações das necessidades” (p. 20). Para identificar as necessidades, Gouveia optou pela distribuição hierárquica de Maslow (1954) e, a partir daí, procedeu à identificação e operacionalização dos valores e grupos de valores que constituem o seu modelo. Gouveia postula que os valores se organizam em torno de três critérios de orientação - pessoal, central e social -, cada um subdividido em duas funções psicossociais: experimentação e realização, existência e suprapessoal, interacional e normativa, respectivamente.

O modelo de Schwartz foi elaborado progressivamente (Schwartz, 1992, 1994, 2005b; Schwartz \& Bilsky, 1987; Schwartz \& Bilsky, 1990) por meio de um diálogo entre a teoria e a realidade observada. A partir do tipo de objetivo ou motivação que expressa cada valor, o autor postulou 10 tipos motivacionais que abrangem o conjunto de valores identificados nas diversas culturas. Esses tipos motivacionais tendem a ser universais e apresentam uma estrutura dinâmica de relações de congruência e de conflito. Os 10 tipos motivacionais são os seguintes: Autodeterminação, Estimulação, Hedonismo, Realização, Poder, Segurança, Conforto, Tradição, Benevolência e Universalismo (ver Figura 1). Várias características desse modelo devem ser salientadas. Em primeiro lugar, o modelo associa intimamente os valores com as motivações subjacentes, de tal forma que a estrutura dos valores transforma-se na estrutura da motivação humana. Schwartz (2005b) afirma: “O que proponho aqui é uma teoria unificadora para o campo da motivação humana, uma maneira de organizar as diversas necessidades, motivos e objetivos propostos em outras teorias" (p. 21). Em segundo lugar, a estrutura circular (ver Figura 1) do modelo representa a dinâmica das relações de congruência e de conflito entre os tipos motivacionais. Assim, "quanto mais próximos dois tipos motivacionais estão em qualquer uma das direções ao 


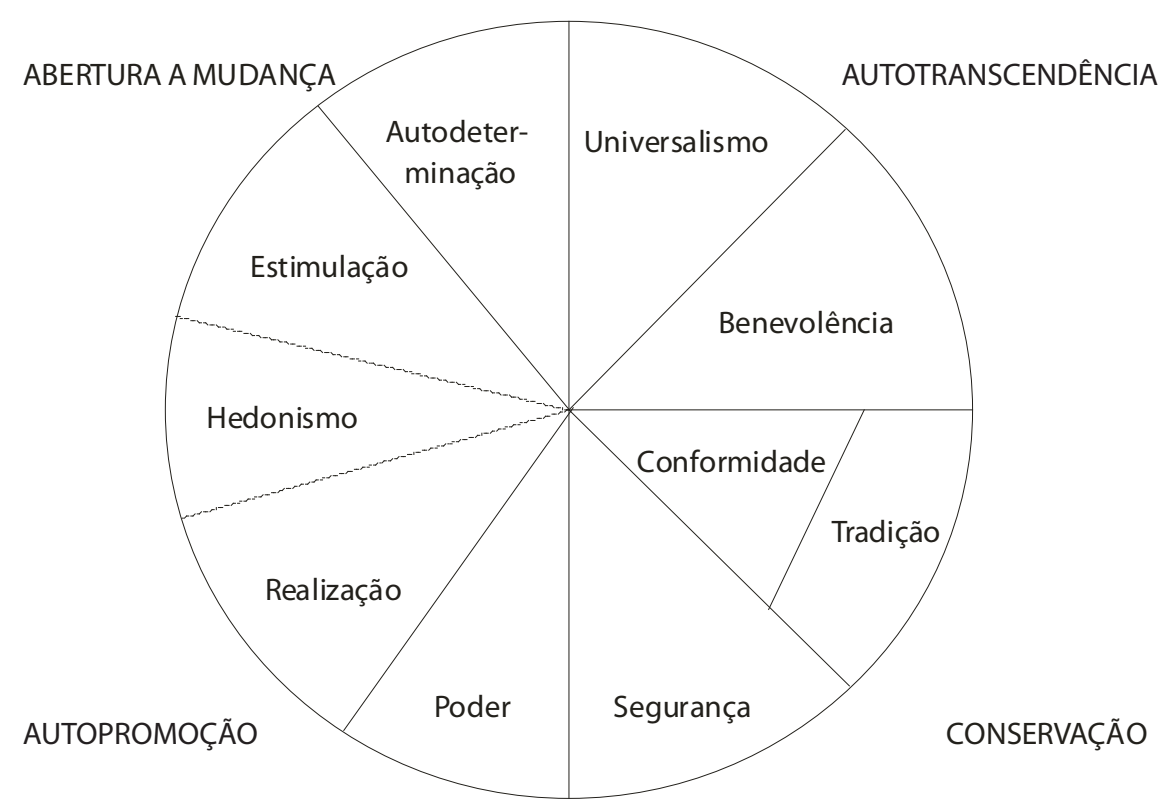

Figura 1. Estrutura dos Valores Humanos segundo Schwartz (2005b)

redor do círculo, mais semelhantes são suas motivações subjacentes. Quanto mais distantes, mais antagônicas são suas motivações subjacentes" (Schwartz, 2005b, p. 29). Em terceiro lugar, a estrutura circular expressa também a continuidade do conteúdo motivacional entre os tipos motivacionais e entre os próprios valores. Dessa forma, o modelo postula que os valores situam-se ao longo de um continuum motivacional. Finalmente, os 10 tipos motivacionais integram-se em duas dimensões bipolares: Abertura à mudança versus Conservação e Autotranscendência versus Autopromoção (ver Figura 1). Os pólos dessas duas dimensões constituem os chamados fatores de ordem superior.

O modelo foi verificado por meio de pesquisa transcultural em 67 países, com uma amostra total de 64.271 (Schwartz, 2005b). Para a verificação do modelo, o autor construiu dois instrumentos: o Schwartz Values Survey (SVS) e o Portrait Values Questionnaire (PVQ). Esses instrumentos têm sido traduzidos para mais de 40 línguas e são amplamente utilizados em pesquisas nacionais e internacionais.

O Schwartz Values Survey (SVS) apresenta grandes semelhanças com o RVS, mas também muitas divergências. Schwartz (1992, 1994, 2005b) considera que existe um número ilimitado de valores, mas que esses podem ser organizados em um número limitado de tipos motivacionais de valores. A partir desse pressuposto, o autor elaborou o modelo teórico acima descrito. O SVS foi construído a partir desse modelo. Os itens foram selecionados a priori para expressar os diversos aspectos do objetivo motivacional de cada um dos tipos motivacionais. Foram aproveitados vários itens do RVS e outros foram elaborados especificamente para o SVS. A estrutura dos itens do SVS é a mesma que a dos itens do RVS: cada um dos valores proposto é seguido por uma frase explanatória, entre parênteses. Os 57 itens do instrumento foram divididos em duas listas correspondentes aos valores terminais e instrumentais. Contrariamente ao RVS, que é de tipo ordinal, o SVS utiliza uma escala que permite à pessoa expressar a importância que possui para ela cada um dos valores, como um princípio que orienta a sua vida. A escala é de nove pontos e varia de -1 (oposto aos meus valores) a 7 (suprema importância). Schwartz (2005b) justifica a escala utilizada da seguinte forma:

Essa escala não simétrica é esticada no extremo superior e condensada no extremo inferior porque a pré-testagem demonstrou que tal escala mapeia a maneira com que as pessoas pensam nos valores, vendo a maioria deles como variando entre medianamente e muito importantes. A escala também possibilita que os sujeitos relatem sua oposição a valores que eles tentam expressar ou promover. (p. 31-32)

A validação do instrumento foi realizada, progressivamente, com 210 amostras provenientes de 67 países, com um total de 64.271 sujeitos. Foi realizada a SSA para cada uma das amostras. Os resultados revelaram que cada um dos tipos motivacionais propostos formou, na quase totalidade das amostras, uma região distinta (Schwartz, 2005b). A validação do SVS para o Brasil foi realizada por Tamayo e Schwartz (1993).

Apesar da evidência fornecida pela forte convergência dos resultados nas numerosas amostras estudadas, ficaram algumas preocupações relativas à validação do SVS, no sentido de que a SSA deixa uma indeterminação estatística já que não fornece indicações objetivas e precisas sobre a orientação e a localização dos eixos que organizam os grupos de valores que constituem os tipos motivacionais. Dessa forma, o pesquisador, ao traçar os eixos, pode seguir o caminho mais favorável para a verificação do modelo. Para contornar essa dificuldade, Schwartz e Boehnke (2004) realizaram análise fatorial confirmatória com dados de 23 países e Gouveia e cols. (2001) o fizeram com uma amostra espanhola de 477 sujeitos. No primeiro artigo, os resultados deram apoio ao modelo teórico proposto, inclusive das relações de conflito entre os tipos motivacionais. A pesquisa de Gouveia e cols. ofereceu apoio parcial ao modelo de Schwartz (1992) já que as relações de conflito não foram confirmadas e os índices de ajuste foram moderados. Tais divergências devem-se em especial à diferença de técnicas utilizadas. Diferentemente 
de Gouveia e cols. (2001), que utilizaram o modelo padrão de equações estruturais, Schwartz e Boehnke (2004) desenvolveram um modelo específico para a análise do modelo proposto teoricamente.

Para superar o caráter abstrato característico do SVS, o que dificulta a sua utilização com crianças e com pessoas de baixo nível de escolarização, Schwartz e cols. (2001) construíram, com base no mesmo modelo dos 10 tipos motivacionais (cf. Figura 1), mais um instrumento para avaliação dos valores, o Portrait Values Questionnaire (PVQ). A versão inicial do PVQ constava de 29 itens. A versão atual está composta por 40 itens, cada item representa um valor presente no SVS, tendo sido privilegiado aqueles valores com significado similar entre culturas. Os itens consistem em curtas descrições de pessoas e não expressam diretamente valores, mas objetivos, desejos e aspirações de pessoas. Essas descrições apontam implicitamente para a importância dada pela pessoa aos diversos valores. Para cada item, o sujeito deve responder: "Quanto essa pessoa se parece com você?". As alternativas de resposta são as seguintes: "Se parece muito comigo", "Se parece comigo", "Se parece mais ou menos comigo", "Se parece pouco comigo", "Não se parece comigo”, "Não se parece nada comigo”. Assim, não há uma escala de resposta numérica. O sujeito utiliza somente uma escala de resposta verbal, o que não requer a transformação de seu julgamento em número, reduzindo o seu esforço cognitivo e simplificando sua tarefa. As respostas, porém, são codificadas pelo pesquisador, que atribui a elas valores que variam de cinco a zero, correspondendo, respectivamente, à primeira e à última opção rotulada da escala verbal.

A versão inicial do PVQ foi validada com amostras representativas da África do Sul $(\mathrm{n}=3.210)$ e da Itália $(\mathrm{n}=5.867)$ e com uma amostra de adolescentes de Uganda $(n=840)$. Os resultados da SSA confirmaram, em todas as amostras, os 10 tipos motivacionais propostos no modelo (Schwartz \& cols., 2001). A versão atual do PQV, com 40 itens, foi validada com 14 amostras provenientes de sete países: Alemanha, Chile, Indonésia, Itália, Peru, Polônia e Ucrânia. Os resultados da SSA mostraram que cada um dos 10 tipos motivacionais de valores postulados formou uma região específica ou conjunta com um tipo motivacional adjacente, como prevê a teoria (Schwartz, 2005a).

Pasquali e Alves (2004) tentaram realizar a validação do PVQ. Eles aplicaram a versão brasileira, fornecida pelos autores deste artigo, a uma amostra de 4.000 usuários de telefonia celular. Por meio de análise fatorial exploratória, identificaram quatro fatores primários e dois de segunda ordem. Os autores denominaram os fatores primários de Pacifismo, Dominação, Tradicionalismo e Dinamismo Hedônico. O primeiro dos fatores de segunda ordem foi denominado Paz e Respeito e está constituído pelos itens dos fatores Pacifismo e Tradicionalismo. O segundo fator, Auto-Promoção, está composto por itens dos fatores de Dominação e Dinamismo Hedônico.

A primeira coisa que chama a atenção nessa tentativa de validação do PVQ é a terminologia utilizada por Pasquali e Alves (2004) para nomear os fatores obtidos, terminologia que difere significativamente do modelo circular utilizado para a construção desse instrumento e que os próprios autores apresentam no seu artigo de validação. A mudança de terminologia certamente confunde o leitor, que fica com a convicção de que os autores acharam resultados diferentes dos esperados. Por outro lado, a análise fatorial exploratória não é adequada para a verificação do modelo circular de Schwartz (ver Figura 1), visto que ela não consegue dar conta das relações dinâmicas entre os tipos motivacionais. O modelo teórico do PVQ exige não somente encontrar os tipos motivacionais propostos, mas também a sua adequada localização espacial de forma a garantir a continuidade motivacional entre eles e entre os próprios valores que os compõem, bem como as relações de congruência e antagonismo. Isso explica porque os 10 tipos motivacionais não foram encontrados. Portanto, a validade de construto do PVQ não foi estabelecida. Os resultados limitaram-se a quatro fatores que apresentam certa relação com os fatores de ordem superior, postulados no modelo. Analisando os resultados de Pasquali e Alves (2004), constata-se que os quatro fatores primários replicam, grosso modo, os quatro fatores de ordem superior do modelo de Schwartz (1992) (ver Figura 1). Pacifismo (constituído por itens de benevolência + universalismo + tradição + segurança + autodeterminação) é uma mistura na qual predominam valores de Autotranscendência; Dominação (integra itens de poder + realização) equivale aproximadamente a Autopromoção; Tradicionalismo (composto por itens de tradição + conformidade) equivale aproximadamente a Conservação; e Dinamismo Hedônico (constituído por itens de hedonismo + estimulação) equivale aproximadamente a Abertura à Mudança. Além disso, como era de se esperar, os resultados da análise fatorial não forneceram nenhuma informação sobre a relação de antagonismo entre os pólos das duas dimensões que constituem os fatores de ordem superior postulados no modelo. Finalmente, os fatores de segunda ordem não correspondem a nenhum dos componentes teóricos do modelo. Pelo contrário, eles são relativamente opostos aos pressupostos teóricos.

A pesquisa relatada neste artigo teve como objetivo geral validar o PVQ para o Brasil e como objetivos específicos comparar os resultados encontrados com a hierarquia pancultural de valores e comparar os resultados encontrados para diferenças de gênero com as pesquisas de validação do instrumento original. Apesar de existir no país um instrumento validado para avaliação dos valores, o IVS, e de ser bastante utilizado em pesquisa, a validação do PVQ justificase por suas características intrínsecas, por sua adequação para pesquisas com crianças e adolescentes e com pessoas de baixo nível de escolaridade e pela demanda por parte dos pesquisadores. A sua utilidade é tal que, mesmo antes da publicação dos resultados da sua validação, o PVQ já tem sido utilizado em várias pesquisas. Os autores deste artigo forneceram a versão brasileira e os resultados aqui relatados a vários pesquisadores.

\section{Método}

\section{Participantes}

A amostra foi constituída por 614 estudantes, sendo 31,4\% do Ensino Fundamental (a partir da $5^{\mathrm{a}}$ série), 34,4\% do Ensino Médio e 34,2\% do Ensino Superior. Do total de participantes, $51,4 \%$ eram do sexo feminino e a média de idade foi de 18 anos $(\mathrm{dp}=4,73)$, variando de 10 a 35 anos. 


\section{Instrumento}

O Portrait Values Questionnaire (PVQ) é composto por 40 itens que descrevem objetivos, aspirações ou desejos que implicitamente apontam para a importância de um tipo motivacional em uma pessoa (Schwartz, 2005a). Exemplo de uma descrição para representar o valor Criatividade é "Pensar em novas idéias e ser criativa é importante para ela. Ela gosta de fazer coisas de maneira própria e original". A tarefa do respondente é avaliar o quanto a pessoa descrita é parecida consigo, utilizando uma escala de resposta com as seguintes alternativas: Se parece muito comigo; Se parece comigo; Se parece mais ou menos comigo; Se parece pouco comigo; Não se parece comigo; Não se parece nada comigo. Além da escala, foram acrescentados dados demográficos para caracterizar a amostra.

Foi utilizado o processo de tradução-retradução da versão em inglês para o português do Brasil. As duas versões em inglês foram comparadas e não foram encontradas diferenças de significado entre elas.

\section{Procedimento}

A coleta de dados foi realizada em duas escolas públicas de Ensino Fundamental e Médio e em uma universidade pública da cidade de Brasília, no ano de 2002. Após aprovação da escola, os pesquisadores se apresentavam em sala de aula, explicavam o objetivo da pesquisa e solicitavam a colaboração dos alunos para responderem o questionário.

\section{Análise de dados}

Para validar a estrutura proposta teoricamente, foi utilizado o Escalonamento Multidimensional (MDS) com o método de escalonamento do coeficiente de alienação de Guttman. O MDS é uma técnica de análise multivariada que permite representar as proximidades entre um conjunto de objetos ou estímulos como distâncias em um espaço de baixa dimensionalidade (geralmente duas ou três dimensões). A representação é gráfica e a distância entre os pontos indica o grau de semelhança entre as variáveis. Assim, quanto mais próximos, maior a semelhança entre eles. A técnica busca o número mínimo de dimensões para representar as correlações. Para julgar a adequação do modelo, utiliza-se o coeficiente de alienação (que varia de 0 a 1) e os gráficos de resíduos (Borg \& Groenen, 1997; Shye, Elizur \& Hoffman, 1994). Um modelo aceitável deve alcançar coeficientes de alienação inferiores a 0,20 e o gráfico de resíduos deve ter os seus pontos agrupados ao redor da diagonal, pois valores que se afastam da diagonal indicam resíduos e, portanto, falta de adequação do modelo.

Essa técnica é mais adequada para análise de dados derivados de desenhos de pesquisa que utilizam a análise das facetas (Shye \& cols., 1994), como é o caso da teoria de valores de Schwartz (1992). Além disso, essa é a técnica comumente usada para testar o modelo proposto. Outro aspecto a favor do uso da técnica é que a teoria de valores não pressupõe linearidade de correlações entre os itens, pelo contrário, o modelo é quase-circumplexo (Schwartz \& Boehnke, 2004), ou seja, os valores se localizam ao longo de uma estrutura circular, a força da associação entre as variáveis é mais forte em variáveis próximas e diminui à medida que a distância no círculo aumenta.

Diferentemente da análise fatorial, o MDS permite a distinção de regiões esperadas teoricamente e não o agrupamento identificado meramente por critérios formais. E mais, os fatores da análise fatorial não estão diretamente relacionados às regiões do escalonamento, pois os fatores são dimensões/vetores subjacentes ao agrupamento de variáveis, enquanto as regiões são formadas na superfície dos pontos finais dos vetores. Assim, pode-se esperar $m+1$ fatores em $m$ dimensões de MDS (Borg \& Groenen, 1997).

Os dados foram analisados pelo programa Systat, versão 10. Além disso, foram calculados os coeficientes alpha de Cronbach para analisar a fidedignidade dos tipos motivacionais, bem como as médias, o desvio-padrão e os intervalos de $95 \%$ de confiança das médias das regiões para a amostra. Para comparar as médias entre gênero, foi usada a Análise de Variância (ANOVA).

\section{Resultados e Discussão}

A análise de escalonamento multidimensional em uma estrutura bidimensional indicou um coeficiente de alienação de 0,19 . Esse valor é aceitável até 0,20, segundo Shye, Borg e Hoffman (1994). Entretanto, deve ser observada a coerência teórica dos resultados para garantir a sua adequação.

Os resultados do diagrama de Shepard também foram favoráveis, uma vez que os dados estão ao redor da diagonal, indicando poucos resíduos com valores elevados, conforme observado na Figura 2.

A configuração final apresentada nas análises pode ser visualizada na Figura 3. Observa-se que foram encontradas sete das 10 regiões previstas teoricamente: Universalismo/ Benevolência $(\alpha=0,78)$, Conformidade $(\alpha=0,59)$, Tradição $(\alpha=0,47)$, Segurança $(\alpha=0,54)$, Poder/Realização $(\alpha=0,77)$, Autodeterminção/Hedonismo $(\alpha=0,68)$ e Estimulação $(\alpha=0,50)$. Os tipos motivacionais Autodeterminação e Hedonismo, Universalismo e Benevolência, bem como Poder e Realização não se diferenciaram. No entanto, observa-se que esses tipos motivacionais compartilham aspectos motivacionais semelhantes: no primeiro caso, ambos pertencem a Abertura à Mudança; no segundo, ambos pertencem a Autotranscendência; e, no último, ambos pertencem a Autopromoção. Essa forma de agregação é comum em análises desse tipo e tem sido encontrada em diversas amostras pesquisadas por Schwartz (2005b). Por exemplo, em 36\% das amostras por ele pesquisadas, Universalismo e Benevolência formaram uma região conjunta. Entretanto, Schwartz (1992) aponta que os tipos motivacionais Poder, Realização e Hedonismo haviam se diferenciado em todas as amostras, o que não ocorreu na amostra brasileira.

$\mathrm{O}$ fato dos tipos motivacionais se misturarem significa que eles compartilham bastante a motivação subjacente. Como propõe a teoria, os valores formam contínuos e a divisão em regiões é arbitrária. Assim, em dados reais, é comum haver esse colapso entre tipos adjacentes. O que indicaria rejeição 
Tabela 1. Dados descritivos do presente estudo e da pesquisa de Schwartz (2005a).

\begin{tabular}{|c|c|c|c|c|c|c|c|}
\hline \multirow{3}{*}{ Média } & \multicolumn{3}{|c|}{ Presente Pesquisa } & & \multicolumn{3}{|c|}{ Schwartz (2005a) } \\
\hline & \multirow{2}{*}{ Desvio-padrão } & \multicolumn{2}{|c|}{ Intervalo de confiança 95\% } & & \multirow{2}{*}{ Hierarquia } & \multirow{2}{*}{ Média } & \multirow{2}{*}{ Hierarquia } \\
\hline & & máximo & mínimo & & & & \\
\hline 3,97 & 0,72 & 4,02 & 3,91 & 1 & $\begin{array}{l}\text { Universalismo/ } \\
\text { Benevolência }\end{array}$ & $4,22 / 4,30$ & $3 / 2$ \\
\hline 3,71 & 0,84 & 3,77 & 3,64 & 2 & $\begin{array}{c}\text { Hedonismo/ } \\
\text { Autodeterminação }\end{array}$ & $3,94 / 4,41$ & $4 / 1$ \\
\hline 3,46 & 0,85 & 3,55 & 3,40 & 3 & Segurança & 3,88 & 5 \\
\hline 3,34 & 1,05 & 3,43 & 3,26 & 4 & Estimulação & 3,84 & 6 \\
\hline 3,25 & 0,92 & 3,18 & 3,33 & 5 & Conformidade & 3,55 & 8 \\
\hline 2,95 & 1,02 & 3,03 & 2,87 & 6 & Tradição & 3,05 & 9 \\
\hline 2,50 & 1,05 & 2,58 & 2,42 & 7 & Poder/Realização & $2,96 / 3,80$ & $10 / 7$ \\
\hline
\end{tabular}

das hipóteses seria o colapso de tipos opostos. Com relação a esse aspecto, os resultados apóiam a teoria dado que os opostos formaram regiões distintas.

Com relação à ordem encontrada para os tipos motivacionais, Estimulação e Benevolência aparecem em disposição diferente da prevista teoricamente. Assim, para corrigir os erros na configuração encontrada, seriam necessários cinco movimentos ${ }^{2}$ : três movimentos para separar Poder de Realização, Universalismo de Benevolência e Hedonismo de Autodeterminação, e mais dois movimentos para adequar Benevolência e Estimulação. Segundo Schwartz (2005a), qualquer arranjo que requer menos de sete movimentos difere significativamente $(p<0,05)$ de um arranjo randômico na direção da estrutura prototípica (com menos de cinco movimentos, $p<0,01)$. Nas amostras de Schwartz (2005b), a média de movimentos para todas as amostras foi de 1,79 , bastante inferior à encontrada no presente estudo. O resultado aqui encontrado, com desvio acima da média, pode ser devido a desvios randômicos, ou talvez à idade dos respondentes que, por serem muito jovens, ainda não têm uma estrutura de valores solidamente formada. Outras amostras nacionais deverão ser investigadas para confirmar esses resultados. Apesar disso, os dados dão apoio ao modelo tanto no que se refere aos tipos motivacionais e à sua localização espacial, quanto às relações de congruência e antagonismo (ver Figura $3)$. Os resultados, portanto, apresentam indícios positivos que apontam para a validade de construto do QPV. Os pesquisadores e profissionais interessados no uso da escala podem obter

2 Schwartz (2005b) define movimentos como "o número de inversões simples na ordem dos tipos motivacionais adjacentes que são necessárias para rearranjar a ordem observada para adequar-se com a ordem prototípica... Nos casos em que os tipos motivacionais formaram uma região conjunta, essa região teve de ser separada. Se os itens de tipos motivacionais diferentes estavam totalmente misturados, essa separação contou como um movimento. Se os itens de cada tipo motivacional estavam predominantemente no lado previsto da região conjunta, a separação contou como meio movimento". (p.39) diretamente com a segunda autora deste artigo o questionário e os itens que compõem cada tipo motivacional.

A convergência com parâmetros e resultados obtidos com outras amostras em diversos lugares do mundo reforça a evidência empírica em relação à validade do QPV. Os escores médios obtidos com essa amostra são apresentados junto com os obtidos com o QPV por Schwartz (2005a) em uma amostra de 2.483 estudantes de sete países (Chile, Alemanha, Indonésia, Itália, Peru, Polônia e Ucrânia). Na Tabela 1, observa-se que a hierarquia dos tipos motivacionais encontrada nesta pesquisa converge, em linhas gerais, com a hierarquia encontrada por Schwartz (2005a), exceto pelo fato de Realização ocupar um lugar mais baixo na hierarquia brasileira. Talvez essa diferença se deva ao fato de os fatores Poder e Realização se colapsarem no presente estudo; entretanto, mesmo considerando a média conjunta desses

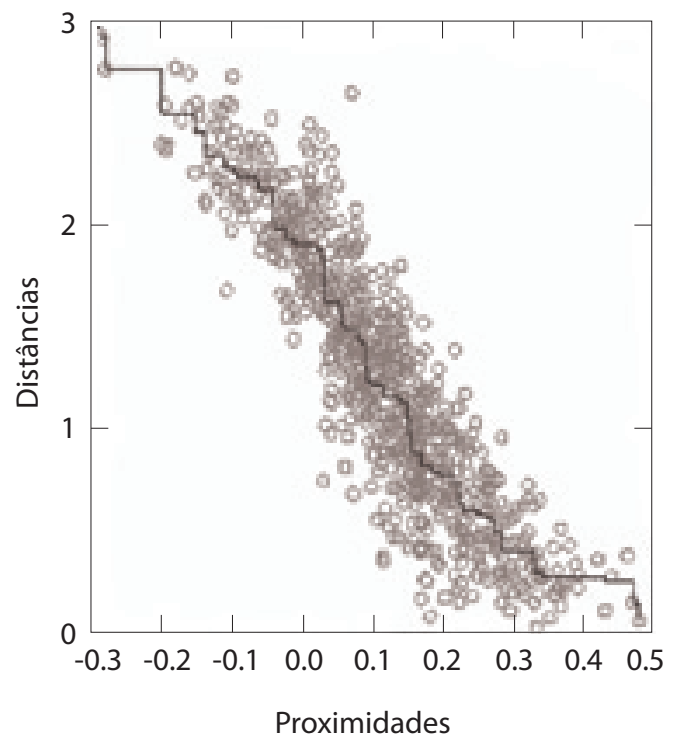

Figura 2. Diagrama de Shepard. 


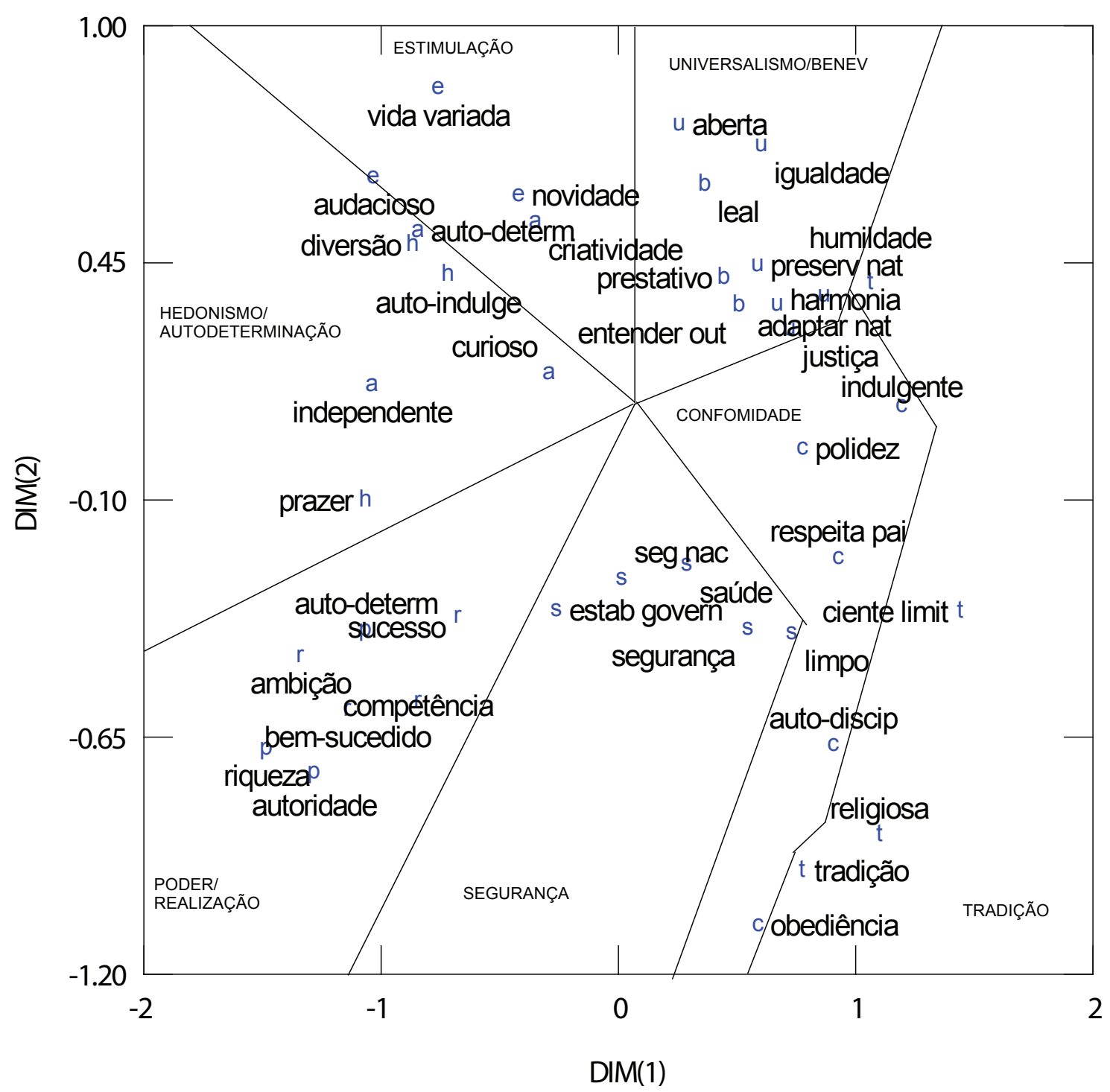

Figura 3. Escalonamento multidimensional do Questionário de Perfis de Valores. As letras indicam a classificação teórica dos itens pelos tipos motivacionais ( $\mathrm{u}=$ universalismo, $\mathrm{b}=$ benevolência, $\mathrm{c}=$ conformidade, $\mathrm{t}=$ tradição, $\mathrm{s}=$ segurança, $\mathrm{r}=\mathrm{realização,} \mathrm{p}=$ poder, $\mathrm{h}=$ hedonismo, $\mathrm{a}=$ autodeterminação, e=estimulação).

dois tipos motivacionais na amostra de Schwartz (2005a), a posição na hierarquia da amostra brasileira é mais baixa.

Os resultados desta pesquisa concordam também com a hierarquia pancultural dos tipos motivacionais. Schwartz e Bardi (2001), utilizando como instrumento de medida o SVS, estabeleceram, com amostras nacionais ou regionais de 13 países, a hierarquia pancultural. Segundo os autores, essa hierarquia permite uma base de comparação para qualquer hierarquia proveniente de qualquer amostra. Os autores acharam que Benevolência, Universalismo e Autodeterminação são consistentemente mais importantes e Tradição e Poder consistentemente menos importantes. A hierarquia apresentada na Tabela 1, obtida com o QPV, confirma que os tipos motivacionais mais e menos importantes são os mesmos que os da hierarquia pancultural estabelecida a partir do SVS. Os resultados da pesquisa relatada neste artigo convergem para os achados internacionais.

Com relação à influência do gênero, as médias para os tipos motivacionais entre homens e mulheres foram com- paradas por meio da ANOVA. Os resultados apontaram diferença significativa para Universalismo/Benevolência $\left(\mathrm{F}_{(561,1)}=12,52, p<0,01\right)$ e Poder/Realização $\left(\mathrm{F}_{(561,1)}=3,90\right.$, $p<0,05)$. As mulheres apresentam médias mais altas para Universalismo/Benevolência [mulheres $=4,09(\mathrm{dp}=0,62)$ e homens $=3,88(\mathrm{dp}=0,77)]$, enquanto os homens apresentam médias mais altas para Poder/Realização [mulheres $=2,43$ $(\mathrm{dp}=1,03)$ e homens $=2,60(\mathrm{dp}=0,99)]$. Esses resultados apóiam parcialmente os achados de Tamayo e Schwartz (1993), pois esses autores encontraram as mesmas diferenças em relação a Universalismo e Benevolência, mas não em relação a Poder e Realização. Por outra parte, Schwartz (2005a), em pesquisas realizadas em diversos países, tanto com o SVS quanto com o QPV, encontrou que mulheres enfatizam mais a Benevolência que os homens e que estes dão mais importância a Poder do que as mulheres. Segundo o autor, este é um "achado consistente" (p. 78) nos países estudados e está em sintonia com as teorias psicanalíticas, feministas culturais, evolucionistas e do papel social que afirmam que 
as mulheres são mais preocupadas com os relacionamentos interpessoais e os homens com a autonomia. Essas diferenças de orientação se expressam em prioridades axiológicas diferentes para homens e mulheres, sendo as mulheres mais voltadas para Benevolência e os homens para Poder.

\section{Comentários Finais}

O objetivo deste estudo foi validar o QVP. Para tanto, foi utilizada a técnica de escalonamento multidimensional que parece mais adequada para testar o modelo teórico.

Esse objetivo foi alcançado e os resultados apontam para a validade de construto do QVP. A convergência de resultados descritivos e comparativos para achados de pesquisas internacionais constitui também um conjunto de elementos importantes de que o instrumento mede o que pretende medir. Ressaltam-se duas limitações do estudo. A primeira refere-se aos índices de confiabilidade que foram baixos, indicando a necessidade de aprimoramento de alguns tipos motivacionais. Em segundo lugar, a amostra utilizada foi de estudantes do Ensino Fundamental, Ensino Médio e Ensino Superior. Apesar dessa escolha ter sido intencional, com o objetivo de averiguar o nível de compreensão do questionário em pessoas com escolaridade mais baixa, para o seu uso amplo é necessária a replicação em amostras de participantes com maior idade e nível de escolaridade mais alto para investigar a validade do instrumento com outros públicos.

\section{Referências}

Allport, G. W., Vernon, P. E., \& Lindzey, G. (1951). A study of values: A scale of measuring the dominant interests in personality. Boston: Houghton Mifflin.

Allport, G. W., Vernon, P. E., \& Lindzey, G. (1970). Study of values: Manual of directions. Cambridge: Houghton Mifflin.

Anderson, N. H. (1968). Likableness ratings of 555 personalitytraits words. Journal of Personality and Social Psychology, 9, 272-279.

Borg, I., \& Groenen, P. (1997). Modern multidimensional scaling: Theory and applications. Nova York: Springer-Verlag.

Duffly, E. (1940). A critical review of investigations employing the Allport-Vernon study of values and others tests of evaluative attitude. Psychological Bulletin, 37, 597-612.

Gouveia, V. V. (2003). A natureza motivacional dos valores humanos: evidências acerca de uma nova tipologia. Estudos de Psicologia (Natal), 8, 431-443.

Gouveia, V. V., Martínez, E., Meira, M., \& Milfont, T. L. (2001). A estrutura e o conteúdo universais dos valores humanos: análise fatorial confirmatória da tipologia de Schwartz. Estudos de Psicologia (Natal), 6, 133-142.

Huntley, C. W., \& Davis, F. (1983). Undergraduate studies of values scores as predictors of occupation 25 years later. Journal of Personality and Social Psychology, 45, 148-155.

Larcebeau, S. (1974). Questionnaire d'intérêts et de valeurs $(Q I V)$. Paris: Service de recherche et de INETOP.
Maslow, A. H. (1954). Motivation and personality. New York: Harper.

McLaughlin, B. (1965). Values in behavioral science. Journal of Religion and Health, 4, 258-279.

Pasquali, L., \& Alves, A. R. (2004). Validação do Portraits Questionnaire - PQ de Schwartz para o Brasil. Avaliação Psicológica, 3, 73-82.

Rokeach, M. (1967). Value Survey. Sunnyvale, California: Halgren Tests.

Rokeach, M. (1973). The nature of human values. New York: Free Press.

Schwartz, S. H. (1992). Universals in the content and structure of values: Theoretical advances and empirical tests in 20 countries. Em M. P. Zanna (Ed.), Advances in experimental social psychology, Vol. 24 (pp. 1-65). San Diego: Academic.

Schwartz, S. H. (1994). Are there universal aspects in the structure and contents of human values? Journal of Social Issues, $50,19-45$.

Schwartz, S. H. (2005a). Validade e aplicabilidade da teoria de valores. Em A. Tamayo \& J. B. Porto (Eds.), Valores e comportamento nas organizações (pp. 56-95). Petrópolis: Vozes.

Schwartz, S. H. (2005b). Valores humanos básicos: seu contexto e estrutura inter-cultural. Em A. Tamayo \& J. B. Porto (Eds.), Valores e comportamento nas organizações (pp. 21-55). Rio de Janeiro: Vozes.

Schwartz, S. H. \& Bardi, A. (2001). Value hierarchies across cultures: Taking a similarities perspective. Journal of Cross Cultural Psychology,32, 268-290.

Schwartz, S. H., \& Bilsky, W. (1987). Toward a universal psychological structure of human values. Journal of Personality and Social Psychology, 53, 550-562.

Schwartz, S. H., \& Bilsky, W. (1990). Toward a theory of the universal content and structure of values: Extensions and crosscultural replications. Journal of Personality and Social Psychology, $58,878-891$.

Schwartz, S. H., \& Boehnke, K. (2004). Evaluating the structure of human values with confirmatory factor analysis. Journal of Research in Personality, 38, 230-255.

Schwartz, S. H., Melech, G., Lehmann, A., Burgess, S., Harris, M., \& Owens, V. (2001). Extending the cross-cultural validity of the theory of basic human values with a different method of measurement. Journal of Cross Cultural Psychology, 32, 519542.

Shye, S., Elizur, D., \& Hoffman, M. (1994). Applied social research methods series, Vol. 35. Introduction to facet theory: Content design and intrinsic data analysis in behaviorl research. Londres: Sage.

Spranger, E. (1931). Types of men. Halle: Niemeyer.

Tamayo, A., \& Schwartz, S. H. (1993). Estrutura motivacional dos valores. Psicologia: Teoria e Pesquisa, 9, 329-348.

Recebido em 29.03.07

Primeira decisão editorial em 20.01.09

Versão final em 13.07.09

Aceito em 14.07.09 